\title{
An overview on the Prus-Szczepanik condition
}

\author{
Carlos A Hernández-Linares, Enrique Llorens-Fuster*, Eva M Mazcuñán-Navarro and Omar Muñiz-Pérez
}

${ }^{*}$ Correspondence:

enrique.llorens@uv.es

Faculty of Mathematics, University

of Valencia, 46100 Burjassot,

Valencia, Spain

\begin{abstract}
In 2005 Prus and Sczcepanik introduced a large class of Banach spaces with the fixed point property for nonexpansive mappings. We say that this class satisfies the PSz condition. Checking that a given Banach space belongs to this class is not an easy task. Here we study the relationship between the PSz condition and other well-known geometrical properties of Banach spaces, and we give easier sufficient conditions for a Banach space to satisfy it.
\end{abstract}

Keywords: fixed point property; E-convexity; (PS) condition; Opial modulus; uniformly noncreasy Banach spaces

\section{Introduction}

Metric fixed point theory is a branch of fixed point theory concerning methods and results that involve properties of an isometric nature. Around 1922, Banach formulated his famous contraction principle, which is considered as the origin of this theory. Since the mid-1960s the outgrowth of a rich and symbiotic interaction between the geometry of Banach spaces and many developments in metric fixed point theory took place. Building on several initial and independent results by Browder, Göhde and Kirk, we now have a rich, though still far from complete, fixed point theory for nonexpansive and related types of mappings in the setting of some classes of Banach spaces.

The classical theory produced a wide range of geometric/topological properties of $\mathrm{Ba}$ nach spaces which were sufficient to ensure the nonexpansive selfmappings of weakly compact convex subsets have fixed points. (See, for instance, [1,2].) Several of these properties are related with the rotundity of the unit ball of the space under consideration, as for instance, the uniform convexity, the uniform smoothness and the uniform nonsquareness. Other relevant properties in this field concern the behavior of the norm of the weak convergent sequences. In this way, in 2005, Prus and Szczepanik (see [3]) defined a large class of Banach spaces, which they called nearly uniformly noncreasy (NUNC for short). This class properly contains the uniformly convex and the uniformly smooth spaces, as well as many of its generalizations.

In the same paper [3] a deep fixed point theorem for nonexpansive mappings was given. We will refer to the assumptions of this theorem as the Prus and Szczepanik condition (PSz in short). In particular, NUNC Banach spaces, among many others, satisfy the PSz condition.

@2014 Hernández-Linares et al.; licensee Springer. This is an Open Access article distributed under the terms of the Creative Commons Attribution License (http://creativecommons.org/licenses/by/2.0), which permits unrestricted use, distribution, and reproduction in any medium, provided the original work is properly cited. 
The aim of this note is to get a better understanding of this property. We will be able to distinguish two nontrivial subfamilies of the class of Banach spaces with the PSz condition, as well as to characterize them in terms of well-known parameters.

Very recently it has been shown in [4] that the so-called $E$-convex Banach spaces, and hence the uniformly nonsquare ones, have the PSz property. Moreover, the relevance of this condition in metric fixed point theory has been reinforced because Betiuk-Pilarska and Wiśnicki in [5] have proved a fixed point theorem for a class of mappings which is larger than the class of nonexpansive mappings, just for Banach spaces satisfying the PSz condition.

\section{Preliminaries}

Throughout this paper we will use the standard notation in Banach space geometry. In particular, if we have a Banach space $(X,\|\cdot\|)$, we denote $B_{X}:=\{x \in X:\|x\| \leq 1\}$ and $S_{X}:=$ $\{x \in X:\|x\|=1\}$. The weak convergence of a sequence $\left(x_{n}\right)$ in $X$ to $x_{0} \in X$ will be denoted $x_{n} \stackrel{w}{\rightarrow} x_{0}$.

For a bounded sequence $\left(x_{n}\right)$ in $X$ we will use the notation

$$
D\left[\left(x_{n}\right)\right]:=\limsup _{n} \limsup _{m}\left\|x_{n}-x_{m}\right\| .
$$

We will often be concerned with the following sets of weakly null sequences:

$$
\mathcal{N}_{X}:=\left\{\left(x_{n}\right): x_{n} \in S_{X}, x_{n} \stackrel{w}{\rightarrow} 0_{X}\right\}
$$

and

$$
\mathcal{M}_{X}:=\left\{\left(x_{n}\right): x_{n} \in B_{X}, D\left[\left(x_{n}\right)\right] \leq 1, x_{n} \stackrel{w}{\rightarrow} 0_{X}\right\}
$$

Note that $\mathcal{M}_{X}$ is always nonempty, and that $\mathcal{N}_{X}$ is nonempty whenever $X$ is not a Schur space.

The following coefficients, defined by Domínguez-Benavides in [6], are useful to get fixed point results:

$$
R(a, X)=\sup \left\{\liminf _{n}\left\|x+x_{n}\right\|:\|x\| \leq a,\left(x_{n}\right) \in \mathcal{M}_{X}\right\}
$$

and

$$
M(X)=\sup \left\{\frac{1+a}{R(a, X)}: a \geq 0\right\} .
$$

A Banach space $X$ is said to have the weak fixed point property for nonexpansive mappings (wFPP for short), if for each nonempty weakly compact convex subset $C$ of $X$, every nonexpansive mapping of $C$ into itself has a fixed point. Nonexpansive mappings are those that have a Lipschitz constant equal to 1.

The weakly convergent sequence coefficient of a Banach space $X$ was introduced by Bynum in 1980 (see [7] for details) and it is defined as

$$
W C S(X):=\inf \left\{\frac{\operatorname{diam}_{\mathrm{a}}\left[\left(x_{n}\right)\right]}{\mathrm{r}_{\mathrm{a}}\left[\left(x_{n}\right)\right]}:\left(x_{n}\right)\right. \text { is a weakly convergent sequence }
$$

which is not norm convergent $\}$. 
Here

$$
\operatorname{diam}_{\mathrm{a}}\left[\left(x_{n}\right)\right]:=\lim _{n \rightarrow \infty}\left(\sup \left\|x_{i}-x_{j}\right\|: i, j \geq n\right)
$$

and

$$
\mathrm{r}_{\mathrm{a}}\left[\left(x_{n}\right)\right]:=\inf \left\{\lim \sup \left\|x_{n}-x\right\|: x \in \overline{\mathrm{co}}\left\{x_{n}: n=1, \ldots\right\}\right\}
$$

It is clear that $1 \leq \operatorname{WCS}(X) \leq 2$.

An important result in [6] is the following.

Theorem 2.1 Every Banach space with $M(X)>1$ has the wFPP.

The following moduli were introduced by Prus and Szczepanik in [3]. Assume now that $X$ fails the Schur property. Then for $x \in X$ and $\varepsilon \geq 0$ put

$$
\begin{aligned}
& d(\varepsilon, x):=\inf _{\left(x_{n}\right) \in \mathcal{N}_{X}} \limsup _{n}\left\|x+\varepsilon x_{n}\right\|-\|x\|, \\
& b(\varepsilon, x):=\sup _{\left(x_{n}\right) \in \mathcal{N}_{X}} \liminf _{n}\left\|x+\varepsilon x_{n}\right\|-\|x\|,
\end{aligned}
$$

and

$$
b_{1}(\varepsilon, x):=\sup _{\left(x_{n}\right) \in \mathcal{M}_{X}} \liminf _{n}\left\|x+\varepsilon x_{n}\right\|-\|x\| .
$$

From the weak lower semicontinuity of the norm, the above moduli are non-negative.

It is straightforward to see that 'limsup' and 'liminf' are interchangeable in the definitions of $d(\varepsilon, x), b(\varepsilon, x)$ and $b_{1}(\varepsilon, x)$.

It is not difficult to see that $R(a, X)$ can be written using the modulus $b_{1}(\cdot, \cdot)$ as follows:

$$
R(a, X)=\sup \left\{b_{1}(1, x)+\|x\|:\|x\| \leq a\right\}
$$

Moreover, from [3, Lemma 4] for any fixed $x \in X$, the functions $d(\cdot, x), b(\cdot, x)$ and $b_{1}(\cdot, x)$ are nondecreasing in the interval $[0,+\infty)$.

In terms of these moduli, in [3] the following class of Banach spaces was introduced.

Definition 2.2 Let $X$ be a Banach space without the Schur property. We say that $X$ is nearly uniformly noncreasy (NUNC for short), if for every $\varepsilon>0$ there is some $t \in(0, \varepsilon]$ such that for every $x \in S_{X}$ it is the case that $d(\varepsilon, x) \geq t$ or $b(t, x) \leq \varepsilon t$. Additionally we treat spaces with the Schur property as being NUNC.

Some widely studied classes of Banach spaces which are relevant in metric fixed point theory are properly included in the class of NUNC Banach spaces. Among many others, one can mention here the so-called uniformly noncreasy Banach spaces, defined by Prus in 1996, as well as several of their generalizations. This notion was introduced to describe a large class of superreflexive Banach spaces with the fixed point property for nonexpansive mappings. Uniformly convex and uniformly smooth Banach spaces are in turn uniformly noncreasy. (See [8] for more details.)

Regarding the wFPP, the main result in [3] is the following. 
Theorem 2.3 Let $X$ be a Banach space failing the Schur property. If there exists $\varepsilon \in(0,1)$ such that for every $x \in S_{X}$ it is the case that $b_{1}(1, x)<1-\varepsilon$ or $d(1, x)>\varepsilon$, then $X$ has the wFPP.

Of course, the NUNC Banach spaces fall within the scope of this theorem. On the other hand, in [9] one can find an example of a superreflexive Banach with the wFPP and lacking the PSz condition.

The following definition is closely inspired on the assumptions of the above theorem, and it has been treated in several recent papers (see $[4,9]$ ).

Definition 2.4 Let $X$ be a non-Schur Banach space. If there exists $\varepsilon \in(0,1)$ such that for every $x \in S_{X}$ it is the case that $b_{1}(1, x)<1-\varepsilon$ or $d(1, x)>\varepsilon$ we say that $X$ satisfies the Prus and Szczepanik condition.

Next we recall several geometrical properties of Banach spaces which are related with the tightest packing of balls of equal size in the unit ball of a Banach space. These properties were introduced by Kottman in [10] and Naidu and Sastry in [11]. For $\eta \in(0,2)$, a subset $A$ of $X$ is said to be symmetrically $\eta$-separated if the distance between any two distinct points of $A \cup(-A)$ is at least $\eta$ and a Banach space $X$ is $O$-convex if the unit ball $B_{X}$ contains no symmetrically $(2-\varepsilon)$-separated subset of cardinality $n$ for some $\varepsilon>0$ and some $n \in \mathbb{N}$. In other words, we have the following.

Definition 2.5 A Banach space $X$ is $O$-convex if there exist $\varepsilon \in(0,1)$ and a positive integer $n \geq 2$ such that for every $x_{1}, x_{2}, \ldots, x_{n} \in S_{X}$, there exist $i, j \in\{1, \ldots, n\}$ with $i<j$ such that

$$
\min \left\{\left\|x_{i}-x_{j}\right\|,\left\|x_{i}+x_{j}\right\|\right\} \leq 2-\varepsilon .
$$

Naidu and Sastry in [11] also characterized the dual property of $O$-convexity. For $\varepsilon>0$, a convex subset $A$ of $B_{X}$ is an $\varepsilon$-flat if $A \cap(1-\varepsilon) B_{X}=\emptyset$. A collection $\mathcal{D}$ of $\varepsilon$-flats is jointly complemented (jcc in short) if, for each distinct $\varepsilon$-flats $A$ and $B$ in $\mathcal{D}$, the sets $A \cap B$ and $A \cap(-B)$ are nonempty. For a positive integer $n$ define

$$
E(n, X)=\inf \left\{\varepsilon>0: B_{X} \text { contains a jcc of } \varepsilon \text {-flats of cardinality } n\right\} .
$$

Definition 2.6 A Banach space $X$ is said to be $E$-convex if $E(n, X)>0$ for some $n \in \mathbb{N}$.

It turns out that a Banach space $X$ is $O$-convex if and only if its dual space $X^{*}$ is $E$-convex, and that $E$-convex Banach spaces are superreflexive.

To end this section, we pay attention to the Opial modulus. It was defined by Prus in [12] (see also [13]), as the function $r_{X}:[0, \infty) \rightarrow \mathbb{R}$ given by

$$
r_{X}(c):=\inf \left\{\liminf _{n}\left\|x+x_{n}\right\|-1:\|x\| \geq c, x_{n} \stackrel{w}{\rightarrow} 0, \liminf _{n}\left\|x_{n}\right\| \geq 1\right\} .
$$

A Banach space $X$ satisfies the uniform Opial condition if and only if $r_{X}(c)>0$ for all $c>0$. Section 3 of Chapter V in [7] is devoted to the study of the uniform Opial condition and the following theorem is proved there. 
Theorem 2.7 The Opial modulus associated to a Banach space satisfies the following properties:

(1) $r_{X}$ is nondecreasing.

(2) $r_{X}(c) \leq$ for all $c>0$.

(3) $r_{X}\left(c_{2}\right)-r_{X}\left(c_{1}\right) \leq \frac{c_{2}\left(c_{2}-c_{1}\right)}{c_{1}}$ for all $c_{2} \geq c_{1}>0$.

(4) If $r_{X}(0)<0$ then $r_{X}$ is constant in $\left[0,-r_{X}(0)\right]$

(5) $r_{X}$ is continuous in $[0,+\infty)$.

Moreover, the Opial modulus is also studied in [13-17], mainly with respect to its relationships with the wFPP.

\section{Two ways to satisfy the PSz condition}

The statement of the Prus and Szczepanik theorem referred to above is built on two inequalities: $b_{1}(1, x)<1-\varepsilon$ and $d(1, x)>\varepsilon$. To clarify the PSz condition we define and study two stronger properties, just paying separated attention to each one of these inequalities.

Definition 3.1 We say that a non-Schur Banach space $X$ has the PSzA condition if there exists $\varepsilon \in(0,1)$ such that for every $x \in S_{X}$ we have $b_{1}(1, x)<1-\varepsilon$ or equivalently

$$
\sup _{x \in S_{X}} b_{1}(1, x)<1
$$

Definition 3.2 When $X$ is a non-Schur Banach space we say that $X$ has the PSzB condition if there exists $\varepsilon \in(0,1)$ such that for every $x \in S_{X}$ we have $d(1, x)>\varepsilon$ or equivalently

$$
\inf _{x \in S_{X}} d(1, x)>0
$$

Remark 3.3 Clearly either the PSzA condition or the PSzB condition implies the PrusSzczepanik condition. In [4, Theorem 3] it is proved that the E-convex Banach spaces satisfy the PSz condition. Indeed, what is really proved there is that these spaces satisfy the PSzA condition. Example 3.3 below shows that the PSz condition does not imply the PSzA condition nor the PSzB condition.

The following is an example of a family of Banach spaces satisfying the PSzA condition, but failing NUNC.

Example 3.1 For $1<p<\infty$ and $\beta \geq \sqrt{2}$ let

$$
X_{p, \beta}:=\left(\mathbb{R} \oplus_{1} E_{\beta}\right) \oplus_{\infty} \ell_{p},
$$

where $E_{\beta}$ is the space $\ell_{2}$ endowed with the norm $|x|_{\beta}:=\max \left\{\|x\|_{2}, \beta\|x\|_{\infty}\right\}$.

For all $\beta \geq 1$ one has $M\left(E_{\beta}\right)>1$. (See [18].) Moreover, from [19] it is well known that the spaces $E_{\beta}$ are $P$-convex for $\beta \geq \sqrt{2}$. Given that the $P$-convexity is preserved under $\ell_{q}$-sums for $1 \leq q \leq \infty$ (see [11]), then the spaces $X_{p, \beta}$ are $P$-convex. Hence, its dual $Y_{p, \beta}:=\left(X_{p, \beta}\right)^{*}$ is $E$-convex, and therefore it satisfies the PSzA condition.

Let us suppose, for a contradiction, that $Y_{p, \beta}$ is NUNC. In this case, from [3, Corollary 8] $X_{p, \beta}$ would be NUNC. However, in [4, Example 4.22] it is shown that $X_{p, \beta}$ fails to satisfy the PSz condition, and hence it cannot be NUNC. 
Remark 3.4 The last example also shows the following:

(a) Condition PSz is not self-dual.

(b) Condition PSzA is not self-dual (one can compare this fact with Corollary 5.6).

(c) P-convexity does not imply the PSz condition.

Example 3.2 Consider the Banach space $E_{\sqrt{2}}$, which consists of the linear space $\ell_{2}$ endowed with the norm

$$
\|x\|_{\sqrt{2}}=\max \left\{\|x\|_{2}, \sqrt{2}\|x\|_{\infty}\right\} .
$$

In [4, Proposition 4.8] it is shown that $E_{\sqrt{2}}$ is not $E$-convex. On the other hand, it is well known (see [8, Theorem 4]) that this space is UNC and hence NUNC (see [3]). This example, along with Example 3.1 shows that no inclusion holds between the classes of $E$-convex and NUNC Banach spaces.

Example 3.3 Consider the Banach space $X=\mathbb{R} \oplus_{1} c_{0}$, that is, the linear space $\mathbb{R} \times c_{0}$ endowed with the norm

$$
\|(t, y)\|=|t|+\|y\|_{c_{0}}
$$

where $t \in \mathbb{R}$ and $y \in c_{0}$. In [3] is proved that $M(X)=1$ and that $X$ is NUNC and hence it has the Prus-Szczepanik condition.

However, $X$ lacks the PSzA condition. In fact, take $x_{0}:=\left(1,0_{c_{0}}\right) \in S_{X}$ and the sequence in $S_{X}$ given by $x_{n}:=\left(0, e_{n}\right)$. Note that

- $\left\|x_{n}-x_{m}\right\|=1$ for $n \neq m$. Hence $D\left[\left(x_{n}\right)\right]=\lim \sup _{n} \lim \sup _{m}\left\|x_{n}-x_{m}\right\|=1$ and $\left(x_{n}\right) \in \mathcal{M}(X)$.

- $x_{n} \stackrel{w}{\rightarrow} 0_{X}$ and $\left\|x_{0}+x_{n}\right\|=1+\left\|e_{n}\right\|_{c_{0}} \equiv 2$.

Then

$$
\begin{aligned}
b_{1}\left(1, x_{0}\right) & =\sup _{\left(y_{n}\right) \in \mathcal{M}(X)} \liminf \left\|x_{0}+y_{n}\right\|-1 \\
& \geq \liminf \left\|x_{0}+x_{n}\right\|-1 \\
& =1 .
\end{aligned}
$$

Thus, $X$ does not have the PSzA condition.

Take $x^{0}=\left(0, e_{1}\right) \in S_{X}$. Let $x_{n}=\left(t_{n}, y_{n}\right)$ be a weak null sequence in $S_{X}$, where $y_{n}=$ $\left(y_{n}(1), y_{n}(2), y_{n}(3), \ldots\right) \in c_{0}$. Then

$$
\begin{aligned}
\underset{n}{\limsup }\left\|x^{0}+x_{n}\right\| & =\limsup _{n}\left(\left|t_{n}\right|+\left\|e_{1}+y_{n}\right\|_{c_{0}}\right) \\
& =\limsup _{n}\left(\left|t_{n}\right|+\max \left\{\left|1+y_{n}(1)\right|,\left|y_{n}(2)\right|,\left|y_{n}(3)\right|, \ldots\right\}\right) \\
& =1 .
\end{aligned}
$$

Hence, for every $x \in S_{X}$,

$$
d(1, x)=\inf _{\left(x_{n}\right) \in \mathcal{N}_{X}}\left\{\liminf _{n}\left\|x+x_{n}\right\|-1\right\} \leq \limsup _{n}\left\|x^{0}+x_{n}\right\|-1=0 .
$$


In consequence $X$ does not have the PSzB condition, although this is a direct consequence of Theorem 6.1 below.

On the other hand, the conditions PSzA and PSzB are compatible.

Example 3.4 Let $H$ be a Hilbert space. For every sequence $\left(x_{n}\right) \in \mathcal{M}_{H}$ one has $\left\langle h, x_{n}\right\rangle \rightarrow 0$ for all $h \in H$. Then

$$
\begin{aligned}
1 & \geq \limsup _{n} \limsup _{m}\left\|x_{n}-x_{m}\right\| \\
& =\limsup _{n} \limsup _{m} \sqrt{\left\|x_{n}\right\|^{2}+\left\|x_{m}\right\|^{2}-\left\langle x_{n}, x_{m}\right\rangle-\left\langle x_{m}, x_{n}\right\rangle} \\
& =\underset{n}{\lim \sup _{\limsup }} \sqrt{\left\|x_{n}\right\|^{2}+\left\|x_{m}\right\|^{2}} \\
& =\sqrt{2} \limsup _{n}\left\|x_{n}\right\| .
\end{aligned}
$$

Thus, $\lim \sup _{n}\left\|x_{n}\right\| \leq \frac{1}{\sqrt{2}}$.

Take $\varepsilon \in\left(0,2-\frac{\sqrt{3}}{\sqrt{2}}\right)$; for $x \in S_{H}$ and $\left(x_{n}\right) \in \mathcal{M}_{H}$ we have

$$
\begin{aligned}
\liminf _{n}\left\|x+x_{n}\right\| & =\liminf _{n} \sqrt{\|x\|^{2}+\left\|x_{n}\right\|^{2}+\left\langle x, x_{n}\right\rangle+\left\langle x_{n}, x\right\rangle} \\
& =\liminf _{n} \sqrt{1+\left\|x_{n}\right\|^{2}} \\
& \leq \frac{\sqrt{3}}{\sqrt{2}} \\
& <2-\varepsilon .
\end{aligned}
$$

Therefore $H$ has the PSzA condition.

Now, consider $\varepsilon \in(0, \sqrt{2}-1)$. For every $x \in S_{H}$ and any sequence $x_{n} \in \mathcal{N}_{H}$ we have

$$
\begin{aligned}
\limsup _{n}\left\|x+x_{n}\right\| & =\limsup _{n} \sqrt{\|x\|^{2}+\left\|x_{n}\right\|^{2}+\left\langle x, x_{n}\right\rangle+\left\langle x_{n}, x\right\rangle} \\
& =\limsup _{n} \sqrt{\|x\|^{2}+\left\|x_{n}^{2}\right\|} \\
& =\sqrt{2}>1+\varepsilon .
\end{aligned}
$$

Consequently $H$ has the PSzB condition.

It is not hard to check that every uniformly convex Banach space also satisfies both PSzA and PszB conditions. However, the following example is a not uniformly convex Banach space which also satisfies these conditions.

Example 3.5 Consider the Banach space $X=\ell_{2} \oplus_{1} \ell_{2}$. In [4] it is showed that $X$ is $E$ convex and hence it enjoys the PSzA condition.

To see that $X$ also enjoys the PSzB condition we need the following lemma, whose proof only needs elementary calculus. 
Lemma 3.5 The restriction of the function $f: \mathbb{R}^{4} \rightarrow \mathbb{R}$ given by $f(a, b, c, d)=\sqrt{a^{2}+c^{2}}+$ $\sqrt{b^{2}+c^{2}}$ to the set $\{(a, b, c, d): a+b=1, c+d=1, a \geq 0, b \geq 0, c \geq 0, d \geq 0\}$ attains its minimum value $\sqrt{2}$ at any point of the form $(a, 1-a, a, 1-a)$ with $a \in[0,1]$.

Take $x=\left(x^{1}, x^{2}\right) \in S_{X}$ and $\left(x_{n}\right)=\left(\left(x_{n}^{1}, x_{n}^{2}\right)\right)$ a weak null sequence in $S_{X}$, then

$$
\begin{aligned}
& \limsup _{n}\left\|x+x_{n}\right\|=\limsup _{n}\left(\left\|x^{1}+x_{n}^{1}\right\|_{2}+\left\|x^{2}+x_{n}^{2}\right\|_{2}\right) \\
& =\limsup _{n}\left(\sqrt{\left\|x^{1}\right\|_{2}^{2}+\left\|x_{n}^{1}\right\|_{2}^{2}+\left\langle x^{1}, x_{n}^{1}\right\rangle+\left\langle x_{n}^{1}, x^{1}\right\rangle}\right. \\
& \left.+\sqrt{\left\|x^{2}\right\|_{2}^{2}+\left\|x_{n}^{2}\right\|_{2}^{2}+\left\langle x^{2}, x_{n}^{2}\right\rangle+\left\langle x_{n}^{2}, x^{2}\right\rangle}\right) \\
& =\limsup _{n}\left(\sqrt{\left\|x^{1}\right\|_{2}^{2}+\left\|x_{n}^{1}\right\|_{2}^{2}}+\sqrt{\left\|x^{2}\right\|_{2}^{2}+\left\|x_{n}^{2}\right\|_{2}^{2}}\right) \\
& \geq \sqrt{2} \quad \text { (from Lemma 3.5). }
\end{aligned}
$$

Thus, $d(1, x):=\inf _{\left(x_{n}\right) \in \mathcal{N}_{X}} \lim \sup _{n}\left\|x+x_{n}\right\|-\|x\| \geq \sqrt{2}-1>0$. Hence $X$ has the PSzB condition.

\section{The PSz condition and $M(X)$}

Next we obtain a characterization of those Banach spaces which satisfy the PSzA condition in terms of the coefficient $M(X)$. First, we recall Lema 4.4 of [5].

Lemma 4.1 Let $X$ be a Banach space. The following conditions are equivalent:

(1) $M(X)>1$,

(2) there exists $a>0$ such that $R(a, X)<1+a$,

(3) for every $a>0, R(a, X)<1+a$.

Moreover, we need the following lemma.

Lemma 4.2 Let $\left(x_{n}\right)$ be a sequence in $B_{X}, x \in X$ such that $\liminf _{n}\left\|a x+x_{n}\right\|>1$ for some $a>0$, then for $b \geq a$ we have

$$
\liminf _{n}\left\|b x+x_{n}\right\| \geq \liminf _{n}\left\|a x+x_{n}\right\| .
$$

Proof There exists $n_{0}$ such that for $n \geq n_{0}$ we have

$$
\left\|a x+x_{n}\right\|>1
$$

Let $f_{n} \in S_{X^{*}}$ such that $f_{n}\left(a x+x_{n}\right)=\left\|a x+x_{n}\right\|$. Then, for $n \geq n_{0}$

$$
f_{n}(x)=\frac{\left\|a x+x_{n}\right\|-f_{n}\left(x_{n}\right)}{a} \geq \frac{\left\|a x+x_{n}\right\|-1}{a}>0 .
$$

Then, for $n \geq n_{0}$

$$
\begin{aligned}
\left\|b x+x_{n}\right\| & \geq f_{n}\left(b x+x_{n}\right)=b f_{n}(x)+f_{n}\left(x_{n}\right) \\
& \geq a f_{n}(x)+f_{n}\left(x_{n}\right)=f_{n}\left(a x+x_{n}\right) \\
& =\left\|a x+x_{n}\right\| .
\end{aligned}
$$


Therefore

$$
\liminf _{n}\left\|b x+x_{n}\right\| \geq \liminf _{n}\left\|a x+x_{n}\right\| .
$$

Proposition 4.3 Let $X$ be a Banach space. If $\left(x_{n}\right)$ is a weakly null sequence in $B_{X}$ and $a \geq 1$, then

$$
\sup _{\|x\| \leq a}\left[\liminf _{n}\left\|x+x_{n}\right\|\right]=\sup _{\|x\|=a}\left[\liminf _{n}\left\|x+x_{n}\right\|\right] .
$$

Proof Since

$$
\sup _{\|x\| \leq a} \liminf _{n}\left\|x+x_{n}\right\| \geq \sup _{\|x\|=a} \liminf \left\|x+x_{n}\right\| \geq a
$$

Then, the equality is clear when $\sup _{\|x\| \leq a} \liminf _{n}\left\|x+x_{n}\right\|=a$.

Suppose that $\sigma:=\sup _{\|x\| \leq a} \liminf _{n}\left\|x+x_{n}\right\|>a$. Choose $\varepsilon \in(0, \sigma-a)$ then there exists $x_{0}$ with $\left\|x_{0}\right\| \leq a$ such that

$$
\liminf _{n}\left\|x_{0}+x_{n}\right\|>\sigma-\varepsilon>a
$$

Note that $x_{0} \neq 0$. Otherwise, we would obtain the following contradiction:

$$
1 \geq \liminf _{n}\left\|x_{n}\right\|=\liminf _{n}\left\|x_{0}+x_{n}\right\|>\sigma-\varepsilon>a \geq 1 .
$$

So, using $\frac{a}{\left\|x_{0}\right\|} \geq 1$ and Lemma 4.2, we have

$$
\liminf _{n}\left\|\frac{a x_{0}}{\left\|x_{0}\right\|}+x_{n}\right\| \geq \liminf _{n}\left\|x_{0}+x_{n}\right\|>\sigma-\varepsilon .
$$

Therefore $\sup _{\|x\|=a} \liminf _{n}\left\|x+x_{n}\right\| \geq \sup _{\|x\| \leq a} \liminf _{n}\left\|x+x_{n}\right\|$.

Theorem 4.4 For a non-Schur Banach space $X$ the following statements are equivalent:

(a) $M(X)>1$,

(b) $R(1, X)<2$,

(c) X has the PSzA condition.

Proof The equivalence between (a) and (b) is an immediate consequence of Lemma 4.1. We claim that for $a \geq 1$,

$$
R(a, X)=\sup _{\|x\|=a} b_{1}(1, x)+a .
$$

Of course,

$$
\begin{aligned}
R(a, X) & =\sup \left\{b_{1}(1, x)+\|x\|:\|x\| \leq a\right\} \\
& \geq \sup \left\{b_{1}(1, x)+\|x\|:\|x\|=a\right\} \\
& =\sup \left\{b_{1}(1, x):\|x\|=a\right\}+a .
\end{aligned}
$$


We are going to see that the previous inequality is in fact an equality. Assume to the contrary that

$$
\sup \left\{b_{1}(1, x)+\|x\|:\|x\| \leq a\right\}>\sup \left\{b_{1}(1, x):\|x\|=a\right\}+a .
$$

Then, there exist $x_{0}$ with $\left\|x_{0}\right\| \leq a$ and $\left(y_{n}\right) \in \mathcal{M}_{X}$ such that

$$
\liminf _{n}\left(\left\|x_{0}+y_{n}\right\|-\left\|x_{0}\right\|\right)+\left\|x_{0}\right\|>\sup \left\{b_{1}(1, x):\|x\|=a\right\}+a .
$$

So, there is a positive number $k$ such that for all $x$ with $\|x\|=a$ we get

$$
\liminf _{n}\left\|x_{0}+y_{n}\right\|>k>b_{1}(1, x)+a \geq \liminf _{n}\left\|x+y_{n}\right\|-\|x\|+a=\liminf _{n}\left\|x+y_{n}\right\| .
$$

Therefore

$$
\liminf _{n}\left\|x_{0}+y_{n}\right\|>k \geq \sup _{\|x\|=a} \liminf _{n}\left\|x+y_{n}\right\|
$$

But from Proposition 4.3 this is a contradiction. So we have

$$
R(a, X)=\sup _{\|x\|=a} b_{1}(1, x)+a .
$$

From the statement of our previous claim, we have

$$
R(1, X)=\sup _{x \in S_{X}} b_{1}(1, x)+1,
$$

which shows the equivalence between (b) and (c).

\section{The PSz condition and Opial modulus}

The aim of this section is to characterize the PSzB condition in terms of the Opial modulus.

Proposition 5.1 For $c \geq 0$,

$$
r_{X}(c)=\inf \left\{\liminf _{n}\left\|x+x_{n}\right\|-1:\|x\| \geq c,\left(x_{n}\right) \in \mathcal{N}_{X}\right\},
$$

and

$$
r_{X}(c) \leq \inf _{\|x\|=c} d(1, x)+c-1
$$

Proof Clearly $r_{X}(c) \leq \inf \left\{\liminf _{n}\left\|x+x_{n}\right\|-1:\|x\| \geq c,\left(x_{n}\right) \in \mathcal{N}_{X}\right\}$.

For $\varepsilon>0$, there exist $x \in X$ and a weak null sequence $\left(x_{n}\right)$ with $\|x\| \geq c$ and $\liminf _{n}\left\|x_{n}\right\| \geq$ 1 such that

$$
\begin{aligned}
r_{X}(c)+\varepsilon & \geq \liminf _{n}\left\|x+x_{n}\right\|-1 \\
& \geq \liminf \left\|x+\frac{x_{n}}{\left\|x_{n}\right\|}\right\|-1 \quad(\text { from [3, Lemma 4]) } \\
& \geq \inf \left\{\liminf _{n}\left\|x+x_{n}\right\|-1:\|x\| \geq c,\left(x_{n}\right) \in \mathcal{N}_{X}\right\} .
\end{aligned}
$$


Moreover,

$$
\begin{aligned}
r_{X}(c) & =\inf _{\|x\| \geq c} \inf _{\left(x_{n}\right) \in \mathcal{N}_{X}} \liminf _{n}\left\|x+x_{n}\right\|-1 \\
& =\inf _{\|x\| \geq c}\left(\inf _{\left(x_{n}\right) \in \mathcal{N}_{X}} \liminf _{n}\left\|x+x_{n}\right\|-\|x\|\right)+\|x\|-1 \\
& =\inf _{\|x\| \geq c} d(1, x)+\|x\|-1 \\
& \leq \inf _{\|x\|=c} d(1, x)+c-1
\end{aligned}
$$

which completes the proof.

Proposition 5.2 Let $X$ be a Banach space. If $\left(x_{n}\right)$ is a weakly null sequence in $B_{X}$ and $c \geq 1$, then

$$
\inf _{\|x\| \geq c}\left[\liminf _{n}\left\|x+x_{n}\right\|\right]=\inf _{\|x\|=c}\left[\liminf _{n}\left\|x+x_{n}\right\|\right]
$$

Proof We have

$$
c \leq \inf _{\|x\| \geq c} \liminf _{n}\left\|x+x_{n}\right\| \leq \inf _{\|x\|=c} \liminf _{n}\left\|x+x_{n}\right\| .
$$

Then, the proposed equality is obvious when $\inf _{\|x\|=c} \liminf _{n}\left\|x+x_{n}\right\|=c$.

Assume that

$$
\inf _{\|x\| \geq c} \liminf _{n}\left\|x+x_{n}\right\|<\inf _{\|x\|=c} \liminf _{n}\left\|x+x_{n}\right\| \quad \text { and } \inf _{\|x\|=c} \liminf _{n}\left\|x+x_{n}\right\|>c .
$$

Then there is some $x_{0} \in X$ with $\left\|x_{0}\right\| \geq c$ such that

$$
\liminf _{n}\left\|x_{0}+x_{n}\right\|<\inf _{\|x\|=c} \liminf _{n}\left\|x+x_{n}\right\|
$$

So, there exists $k>1$ such that

$$
\max \left\{\liminf _{n}\left\|x_{0}+x_{n}\right\|, c\right\}<k<\inf _{\|x\|=c} \liminf _{n}\left\|x+x_{n}\right\| .
$$

Then, for all $x \in X$ with $\|x\|=c$

$$
\max \left\{\liminf _{n}\left\|x_{0}+x_{n}\right\|, c\right\}<k<\liminf _{n}\left\|x+x_{n}\right\| .
$$

In particular

$$
\max \left\{\liminf _{n}\left\|x_{0}+x_{n}\right\|, c\right\}<k<\liminf _{n}\left\|\frac{c x_{0}}{\left\|x_{0}\right\|}+x_{n}\right\|
$$

But from Lemma 4.2 this is a contradiction, because $k>1$ and $\frac{c}{\left\|x_{0}\right\|} \leq 1$. 
Remark 5.3 For the Hilbert space $\ell_{2}$, it is well known that $r_{\ell_{2}}(c)=\sqrt{1+c^{2}}-1$ (see [13]), while

$$
\begin{aligned}
\inf _{\|x\|=c} d(1, x)+c-1 & =\inf _{\|x\|=c} \inf _{\left(x_{n}\right) \in \mathcal{N}(X)}\left\{\lim \sup \left\|x_{n}+x\right\|-\|x\|\right\}+c-1 \\
& =\sqrt{1+c^{2}}-1 .
\end{aligned}
$$

Consequently, in this space, inequality (1) is in fact an equality for every $c \geq 0$. Nevertheless in a general Banach space, we do not know if this holds for $0<c<1$. However, if the Banach space $X$ does not satisfy the uniform Opial condition, then $r_{X}(0)<0$ while $\inf f_{\|x\|=0} d(1, x)+$ $0-1=0$. Since the Opial modulus $r_{X}$ is continuous in $[0, \infty)$, then in this case, equality (2) below fails for $c$ in a nontrivial interval $[0, \alpha)$. Conversely, if $X$ satisfies the uniform Opial condition, then $r_{X}(0)=0$, and hence the equality (2) is satisfied at $c=0$.

Theorem 5.4 $X$ has the PSzB if and only if $r_{X}(1)>0$.

Proof We claim that for $c \geq 1$,

$$
r_{X}(c)=\inf _{\|x\|=c} d(1, x)+c-1
$$

Assume that

$$
r_{X}(c)<\inf _{\|x\|=c} d(1, x)+c-1
$$

Then, there is some $x_{0} \in X$ with $\left\|x_{0}\right\| \geq c$ such that

$$
d\left(1, x_{0}\right)+\left\|x_{0}\right\|-1<\inf _{\|x\|=c} d(1, x)+c-1
$$

Therefore there exists $\left(x_{n}\right) \in \mathcal{N}_{X}$ such that

$$
\liminf _{n}\left\|x_{0}+x_{n}\right\|-\left\|x_{0}\right\|+\left\|x_{0}\right\|<\inf _{\|x\|=c} d(1, x)+c
$$

Thus, there exists $k>0$ such that for all $x \in X$ with $\|x\|=c$ we have

$$
\liminf _{n}\left\|x_{0}+x_{n}\right\|<k<\liminf _{n}\left\|x+x_{n}\right\|-\|x\|+c=\liminf _{n}\left\|x+x_{n}\right\|,
$$

but this is a contradiction with Proposition 5.2.

From our claim, taking $c=1$ we obtain the desired result.

Remark 5.5 In [17] Xu proved that the condition $r_{X}(1)>0$ implies the wFPP. According to the above result, we realize that the Prus-Szczepanik theorem recaptures Xu's result. This condition is relevant to prove some other results in metric fixed point theory as in [15] and [20].

Corollary 5.6 Let $X$ be a reflexive Banach space. Then we have the following.

(a) If $X^{*}$ satisfies PSzB, then $X$ satisfies PSzA.

(b) If $X$ satisfies PSzB, then $X^{*}$ satisfies PSzA. 
Proof From [6, Theorem 3.3] we know that $r_{X^{*}}(1)>0$ implies $M(X)>1$.

Some examples with $r_{X}(1)>0$ are the following.

Example 5.1 (See [13]) For $1<p<\infty$, and $c \geq 0$,

$$
r_{\ell_{p}}(c)=(1+c)^{\frac{1}{p}}-1
$$

Example 5.2 (See [16]) Let us now consider Bynum's spaces $\ell_{p, q}$, which are spaces $\ell_{p}$ endowed with an equivalent norm given by the formula $\|x\|_{p, q}:=\left(\left\|x^{+}\right\|^{q}+\left\|x^{-}\right\|^{q}\right)^{1 / p}$.

If $1<p<\infty$ and $1 \leq q<\infty$ then for all $c \geq 0$,

$$
r_{\ell, q}(c)=\min \left\{\left(1+c^{p}\right)^{\frac{1}{p}}-1,\left(1+c^{q}\right)^{\frac{1}{q}}-1\right\} .
$$

Example 5.3 (See [21]) Let $X_{p}$ be $\ell_{2}$ endowed with the norm

$$
\|x\|:=\left(\left|x_{1}\right|^{p}+\left(\sum_{n=2}^{\infty}\left|x_{n}\right|^{2}\right)^{\frac{p}{2}}\right)^{\frac{1}{p}}
$$

where $p>2$. Then

$$
r_{X_{p}}(c)=\left(c^{p}+1\right)^{\frac{1}{p}}-1
$$

Notice that for all $c \geq 0$,

$$
c-1 \leq r_{X}(c) \leq c .
$$

In particular, $r_{X}(c)>0$ for all $c>1[13]$.

\section{The second way leads to the first one}

Theorem 6.1 If a Banach space $X$ has the PszB condition (equivalently $r_{X}(1)>0$ ), then $X$ has the PszA condition (equivalently $M(X)>1$ ).

Proof From [6, p.841] we know that $M(X) \geq W C S(X)$. On the other hand, in [13] it was shown that $W C S(X) \geq 1+r_{X}(1)$ and the conclusion immediately follows.

Remark 6.2 The above theorem shows that $\mathrm{Xu}$ [17] is, in fact, a particular case of the Domínguez-Benavides fixed point result (Theorem 2.1).

The following example shows that there are spaces with $M(X)>1$ (i.e. satisfying PSzA), but with $r_{X}(1)=0$ (i.e. lacking PSzB).

Example 6.1 The classical Banach space $\left(c_{0},\|\cdot\|_{\infty}\right)$ has the PSzA condition and it fails the PSzB condition.

It is well known (see [6, p.846]) that $M\left(c_{0}\right)=2$, and hence it satisfies the PSzA condition. 
In order to check that $c_{0}$ fails the PSzB condition take $x=e_{1}$ and $x_{n}=e_{n}$ for $n \geq 1$. Then, we have $\|x\|_{\infty} \geq 1, \liminf _{n}\left\|x_{n}\right\|_{\infty} \geq 1$ and $\liminf _{n}\left\|x+x_{n}\right\|_{\infty}=1$. From the definition of the Opial modulus we get

$$
r_{c_{0}}(1) \leq \liminf _{n}\left\|x+x_{n}\right\|_{\infty}-1=0,
$$

and the conclusion now follows from Theorem 5.4.

It is easy to check that $\left(c_{0},\|\cdot\|_{\infty}\right)$ is NUNC. Thus, this property does not imply the condition PSzB.

Competing interests

The authors declare that they have no competing interests.

Authors' contributions

All authors contributed equally and significantly in writing this paper. All authors read and approved the final manuscript.

\section{Acknowledgements}

The authors would like to thank the referees for their valuable comments. The first and the fourth authors have been supported by grants from CONACYT (México). The second and the third authors have been partially supported by grant MTM2012-34847-C02-02 from Spanish Government.

Received: 21 November 2013 Accepted: 29 January 2014 Published: 13 Feb 2014

\section{References}

1. Goebel, K, Reich, S: Uniform Convexity, Hyperbolic Geometry and Nonexpansive Mappings. Dekker, New York (1984)

2. Goebel, K, Kirk, WA: Topics in Metric Fixed Point Theory. Cambridge University Press, Cambridge (1990)

3. Prus, S, Szczepanik, M: Nearly uniformly noncreasy Banach spaces. J. Math. Anal. Appl. 307, 255-273 (2005)

4. Llorens-Fuster, E, Muñiz-Pérez, O: Some relationships between sufficient conditions for the fixed point property. Fixed Point Theory 14, 125-140 (2013)

5. Betiuk-Pilarska, A, Wiśnicki, A: On the Suzuki nonexpansive-type mappings. Ann. Funct. Anal. 4, 72-86 (2013)

6. Domínguez-Benavides, T: Stability of the fixed point property for nonexpansive mappings. Houst. J. Math. 22, 835-849 (1996)

7. Ayerbe-Toledano, J, Domínguez-Benavides, T, López Acedo, G: Measures of Noncompactness in Metric Fixed Point Theory. Operator Theory: Advances and Applications, vol. 99. Birkhäuser, Basel 1997

8. Prus, S: Banach spaces which are uniformly noncreasy. Nonlinear Anal. TMA 30, 2317-2324 (1997)

9. Fetter Nathansky, H, Llorens-Fuster, E: A product space with the fixed point property. Fixed Point Theory Appl. 2012, Article ID 91 (2012)

10. Kottman, CA: Packing and reflexivity in Banach spaces. Trans. Am. Math. Soc. 150, 565-574 (1970)

11. Naidu, SVR, Sastry, KPR: Convexity conditions in normed linear spaces. J. Reine Angew. Math. 297, 35-53 (1976)

12. Prus, S: Spaces with the uniform Opial property. Nonlinear Anal. 18,697-704 (1992)

13. Lin, PK, Tan, KK, Xu, HK: Demiclosedness principle and asymptotic behavior for asymptotically nonexpansive mappings. Nonlinear Anal. 24, 929-946 (1995)

14. Domínguez-Benavides, T, Japón-Pineda, MA: Opial modulus, moduli of noncompact convexity and fixed points for asymptotically regular mappings. Nonlinear Anal. 41, 617-630 (2000)

15. Kuczumow, T: Opial's modulus and fixed points of semigroups of mappings. Proc. Am. Math. Soc. 127, 2671-2678 (1999)

16. Xu, HK: Geometrical coefficients of Banach spaces and nonlinear mappings. In: Recent Advances on Metric Fixed Point Theory. University of Seville, Seville (1996)

17. Xu, HK: Banach space properties of Opial type and fixed point theorems for nonlinear mappings. Ann. Univ. Mariae Curie-Skīodowska, Sect. A LI(2), 25-A, 293-303 (1997)

18. Domínguez-Benavides, T, Japón Pineda, MA: Stability of the fixed point property for nonexpansive mappings in some classes of spaces. Commun. Appl. Nonlinear Anal. 5, 37-46 (1998)

19. Maluta, E: A class of P-convex spaces lacking normal structure. Nonlinear Anal. 75, 2011-2015 (2012)

20. Kuczumow, T, Reich, S: An application of Opial's modulus to the fixed point theory of semigroups of Lipschitzian mappings. Ann. Univ. Mariae Curie-Sk̄odowska, Sect. A LI(2), 17-A, 185-192 (1997)

21. Japón-Pineda, MA: Stability of the fixed point property for nonexpansive mappings. Ph.D. dissertation, University of Seville (1998)

10.1186/1687-1812-2014-37

Cite this article as: Hernández-Linares et al.: An overview on the Prus-Szczepanik condition. Fixed Point Theory and Applications 2014, 2014:37 